\title{
Fano resonance between Stokes and anti-Stokes Brillouin scattering
}

\author{
KwanTo Lai $\odot,{ }^{1}$ Daniel Finkelstein-Shapiro $\odot,{ }^{2}$ Sebastian Lehmann, ${ }^{3}$ Arnaud Devos $\odot,{ }^{4}$ and Pierre-Adrien Mante $\odot^{1,2, *}$ \\ ${ }^{1}$ Department of Applied Physics, The Hong Kong Polytechnic University, Hong Kong SAR \\ ${ }^{2}$ Division of Chemical Physics and NanoLund, Lund University, Lund, Sweden \\ ${ }^{3}$ Division of Solid State Physics and NanoLund, Lund University, Lund, Sweden \\ ${ }^{4}$ Institut d'Électronique, de Microélectronique et de Nanotechnologie, Unité Mixte de Recherche, CNRS 8250, \\ Villeneuve d'Ascq Cedex, France
}

(Received 21 October 2020; revised 1 March 2021; accepted 27 April 2021; published 8 July 2021)

\begin{abstract}
In recent years, the manipulation of Fano resonances in the time domain has unlocked deep insights into a broad spectrum of systems' coherent dynamics. Here, inelastic scattering of light with coherent acoustic phonons is harnessed to achieve complex Fano resonances. The sudden change of phonon momentum during reflection leads to a transition from anti-Stokes to Stokes light scattering, producing two different resonances that interfere in the measurement process. We highlight the conditions necessary to achieve such interference, revealing an underlying symmetry between photons and phonons, and verify the theory experimentally. Then, we demonstrate the possibility to characterize energy and coherence losses at rough interfaces, thus providing a mechanism for nondestructive testing of interface quality. Our results describe numerous unexplained observations in ultrafast acoustics and can be generalized to the scattering of light with any waves.
\end{abstract}

DOI: 10.1103/PhysRevResearch.3.L032010

Fano resonances occur when a broad and featureless continuum of states couples to a discrete resonance with energy lying within the continuum, thereby opening two interfering pathways to reach the continuum manifold from an initial state. It results in an asymmetric line shape in the system's frequency response, characterized by the Fano parameter $q$ [1]. Fano resonances were first studied in the context of photoionization [2] but have since been found in the optical response of metamaterials and plasmonic nanostructures [3-5] and also in mechanical [6], acoustic [7-9], and electronic systems [10]. Interference-based sensors rely on using frequency shifts, more easily measured than amplitude modulations. Therefore Fano resonances are extremely sensitive to perturbations and have been used for characterization and sensing $[3,10,11]$. Furthermore, Fano resonances can also be used to investigate dissipative mechanisms $[12,13]$. The presence of relaxation breaks the time-reversal symmetry and can be described by a complex Fano parameter $[12,14,15]$. By analyzing the trajectories of the $q$ parameter in the complex plane as a function of dissipation strength, dissipation (energy loss) vs decoherence (coherence loss) were successfully distinguished [13].

Fano resonances have also been investigated in the time domain, allowing additional characterization $[16,17]$ and deeper insights into their properties [18,19]. Notably, such studies have shown the coherent nature of magnetoexcitons in GaAs [16] and of electronic wave packets [17]. In the time domain,

\footnotetext{
*pierre-adrien.mante@ chemphys.lu.se

Published by the American Physical Society under the terms of the Creative Commons Attribution 4.0 International license. Further distribution of this work must maintain attribution to the author(s) and the published article's title, journal citation, and DOI.
}

the Fano resonance appears as the interference between a long-lived state (the discrete state) and a short-lived state (akin to the continuum). These time-domain investigations of Fano resonances have showcased the mapping of the Fano parameters $q$ in the frequency domain to a phase $\phi$ in the time domain [18]. A consequence of this relation is the possibility of manipulating Fano resonance using a perturbation that introduces a phase shift in the response of the system's discrete pathway, even when a Fano structure is absent [18]. Such concepts have been used to launch and interrupt a Fano resonance [19] or to observe Fano resonance by inducing simultaneous stimulated emission and absorption [20], and other effects are predicted for these dynamic Fano resonances [21]. Up to now, manipulation of Fano resonances through ultrafast perturbation was limited to atomic systems; however, applying such a concept to condensed matter would unlock vast opportunities for material characterization thanks to the extreme sensitivity of Fano resonance.

In this Letter, we investigate the formation of Fano resonances due to the interaction of light with coherent acoustic phonons (CAPs) reflecting at an interface. Although minima in the measured phonon spectrum had been routinely observed, neither the connection to Fano interferences nor an explanation of the underlying physical mechanism has been provided. We first briefly review the Stokes and anti-Stokes interaction of light with CAPs and highlight the transition from anti-Stokes to Stokes Brillouin scattering during the reflection of CAPs at an interface. During this transition, light undergoes both scatterings simultaneously, leading to interference in the scattering probability, responsible for forming a complex Fano resonance. We perform picosecond ultrasonic experiments on a thin tungsten (W) film that reveals a Fano resonance in the transient reflectivity spectrum in excellent agreement with our model. We finally discuss the possibility of dissociating dissipation from decoherence by tracking the $q$ parameter in 
the complex plane as a function of the laser wavelength, thus enabling nondestructive characterization of the roughness of surfaces and buried interfaces.

In condensed media, Brillouin scattering refers to the scattering of light by waves that modifies the material's refractive index, such as phonons [22], magnons [23], or polaritons [24]. With coherence, additional effects can occur due to the waves' well-defined phase and momentum, such as parametric interactions $[25,26]$ or the possibility to perform time-resolved investigations [27-33]. In the following, we show how we take advantage of the waves' coherent nature to create a Fano response in the scattering of light. We take the example of CAPs, but the results can be generalized to any type of light scattering waves.

We first investigate the scattering of light by CAPs. We carried out picosecond ultrasonic experiments using two different experimental setups. In the first one, we used a Ti:sapphire oscillator, producing 120 -fs optical pulses at a repetition rate of $80 \mathrm{MHz}$, centered at a wavelength tunable between 690 and $1040 \mathrm{~nm}$ in a conventional one-color pump and probe setup at normal incidence to investigate a 110 -nm-thick W thin film deposited on a $\mathrm{Si}$ substrate. At the sample surface, the laser spot size is $20 \mu \mathrm{m}$, and the pump fluence is $2 \mathrm{~mJ} / \mathrm{cm}^{2}$. In the second setup, we used a regeneratively amplified, mode-locked Yb:KGW (ytterbium-doped potassium gadolinium tungstate) based femtosecond laser system operating at $1030 \mathrm{~nm}$ and delivering pulses of $200 \mathrm{fs}$ at a $1 \mathrm{kHz}$ repetition rate. This laser is then used to pump two noncollinearly phase-matched optical parametric amplifiers (NOPAs). The first one generated pump pulses centered at $550 \mathrm{~nm}$ with $35 \mathrm{fs}$ duration. The pump pulses were focused to a diameter of $200 \mu \mathrm{m}$, which corresponds to a laser of fluence of $2 \mathrm{~mJ} / \mathrm{cm}^{2}$. The second NOPA generated probe pulses with about $40 \mathrm{fs}$ pulse duration at wavelengths ranging from 650 to $920 \mathrm{~nm}$. We used this setup to investigate a rough, 100-nm-thick W film deposited on an $\mathrm{Al}_{2} \mathrm{O}_{3}$ substrate. The rough sample was obtained by increasing the gas flux during the chemical vapor deposition of the $\mathrm{W}$ film. To ensure that the rough surface of the $\mathrm{W}$ film does not influence the generation of CAPs, we performed pump-probe experiments with the pump incident on the $\mathrm{Al}_{2} \mathrm{O}_{3} / \mathrm{W}$ interface, while the probe is reflected at the $\mathrm{W} /$ air interface, as described in Fig. 1(a).

For both experimental setups, the absorption of the femtosecond laser pulse leads to the generation of longitudinal CAPs, $\eta(z-v t)$, that propagates in the sample [29,30]. A second time-delayed femtosecond laser pulse, the probe, is Stokes scattered by the CAPs that propagate towards increasing $z$, as depicted in the upper part of Fig. 1(b). This scattering process corresponds to the emission of a phonon at the angular frequency $\Omega_{B}=4 \pi n v / \lambda$, with $n$ being the refractive index at the probe wavelength $\lambda$ and $v$ being the longitudinal sound velocity. The CAPs then propagate to the bottom of the thin film, are reflected towards the surface, and re-enter the probe's penetration region. This time, the probe light is anti-Stokes scattered, corresponding to the absorption of a phonon at the frequency $\Omega_{B}$, as shown in the lower part of Fig. 1(b). During the following reflection of the CAPs, the probe light simultaneously undergoes Stokes and anti-Stokes processes due to the change in phonon momentum. Under certain conditions, the probabilities of Stokes and anti-Stokes

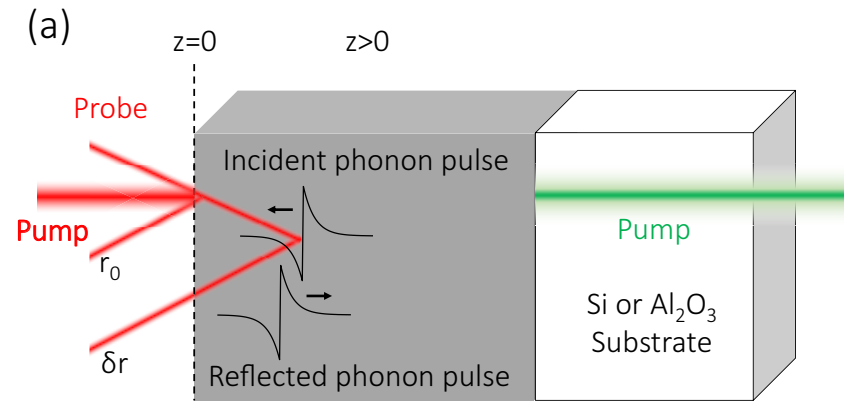

(b)
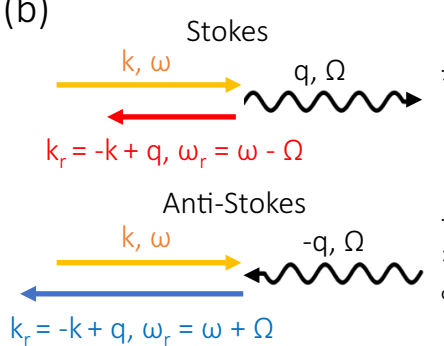

(C) Frequency shift $(\mathrm{GHz})$

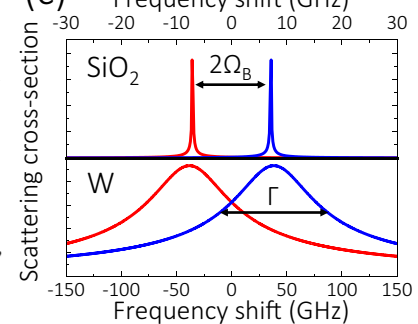

FIG. 1. Principles of light scattering by coherent acoustic phonons. (a) A femtosecond laser (probe) is reflected by a free surface and by a coherent phonon pulse propagating within a semiinfinite substrate and getting reflected at the free surface. (b) Energy and momentum conservation rules before and after the reflection of the phonon pulse. (c) Line shape of Stokes (red) and anti-Stokes (blue) resonance in $\mathrm{SiO}_{2}$ and $\mathrm{W}$. When the material is transparent, the resonances are not overlapping and cannot interfere.

scattering may interfere, leading to the formation of a Fano resonance in the scattering cross section.

We now study the appearance of such Fano resonance. For simplicity in the following, we assume that the reflection of CAPs at the free surface occurs at $t=0$. The timedependent relative reflectance, or scattering cross section, for a monochromatic electromagnetic wave at frequency $\omega$, is given by (see Supplemental Material [34]) [29,32]

$$
\begin{aligned}
\frac{\delta r(t)}{r_{0}}= & \rho e^{-j \phi} \int_{-\infty}^{+\infty} d z \eta(v t-z) \\
& \times\left[H(-z) e^{-2 j k \tilde{n} z}+R H(z) e^{2 j k n \tilde{z}}\right],
\end{aligned}
$$

where

$$
\rho e^{-j \phi}=\frac{4 j k \tilde{n}}{1-\tilde{n}^{2}}\left(\frac{\partial n}{\partial \eta}+j \frac{\partial \kappa}{\partial \eta}\right)
$$

$k$ is the wave vector of the probe light, $R$ is the acoustic reflection coefficient at the free surface, $H(z)$ is the Heaviside function, and $\tilde{n}=n+j \kappa$ and $\frac{\partial n}{\partial \eta}+j \frac{\partial \kappa}{\partial \eta}$ are the complex refractive index and complex photoelastic coefficient at the probe wavelength, respectively. Equation (1) is the convolution of the CAPs with the Green's function of the system, i.e., the light scattering cross section for a $\delta$-like CAP pulse. The first term in the square brackets corresponds to anti-Stokes scattering, and the second corresponds to the Stokes process. At the surface $(z=0)$, the Green's function is discontinuous; that is, the reflection of the CAPs introduces a phase shift.

The scattering cross section $\sigma(\Omega, \omega)$ for photons of frequency $\omega$ by phonons of frequency $\Omega$ is obtained by 
performing the Fourier transform of Eq. (1) (see Supplemental Material [34]):

$$
\sigma(\Omega, \omega)=\frac{\rho e^{-j \phi}}{\Gamma}\left[\frac{1+j \chi}{1+\chi^{2}}+R \frac{1-j \Delta}{1+\Delta^{2}}\right],
$$

where we have introduced $\Gamma=2 \kappa \omega v / c, \chi=\left(\Omega+\Omega_{B}\right) / \Gamma$, and $\Delta=\left(\Omega-\Omega_{B}\right) / \Gamma$. The scattering cross section of Eq. (3) is composed of two resonances corresponding to the Stokes and anti-Stokes scattering. The amplitude of each resonance taken separately is shown in Fig. 1(c) for the case of $\mathrm{SiO}_{2}$ and $\mathrm{W}$ at $820 \mathrm{~nm}$. To calculate these resonances, we take the absolute value of the Stokes or anti-Stokes scattering cross section of Eq. (3), with a refractive index given by $n=1.4606+$ $i 0.0012744$ [35] and a sound velocity of $5.85 \mathrm{~nm} / \mathrm{ps}$ for $\mathrm{SiO}_{2}$ [36], and $n=3.4808+i 2.8435$ [37] and a sound velocity $v=5.2 \mathrm{~nm} / \mathrm{ps}$ for $\mathrm{W}$ [38]. One notices, in Eq. (3), that photons and phonons are the mirror of each other: Looking at the evolution of $\sigma(\Omega, \omega)$ for a fixed $\omega$ and a varying $\Omega$, or vice versa, we obtain two resonances corresponding to the resonant Stokes and anti-Stokes scattering for photons, or the resonant absorption and emission for phonons.

Finally, we can rewrite Eq. (3) as the sum of a complex Lorentzian and a complex Fano resonance (see Supplemental Material [34]):

$$
\sigma(\Omega, \omega)=\frac{\rho e^{-j \phi}}{\Gamma(1-j \chi)}\left[\frac{(\Delta+q)^{2}+(1+j q)^{2}}{1+\Delta^{2}}\right],
$$

where $q=-R(j+\chi) / 2$ is the Fano parameter. The Fano resonance is thus resulting from the interference between the Stokes and anti-Stokes scattering of light.

By analyzing the Fano parameter, we can achieve deeper insights into the phenomenon. We see that the real part, $-R \chi / 2$, is proportional to $n / \kappa$ in the vicinity of the resonance (see Supplemental Material [34]) and represents the overlap between the Stokes and anti-Stokes resonances shown in Fig. 1(c). When $n$ increases, the Brillouin scattering frequency increases, reducing the overlap between Stokes and antiStokes. This leads to a disappearance of the Fano resonance as $q \rightarrow \infty$. Moreover, when $\kappa$ increases, the penetration of light is smaller, which broadens Brillouin resonances and increases the overlap between Stokes and anti-Stokes resonances. In Fig. 1(c), we can see how the absorption impacts the overlap of resonance. $\mathrm{In}_{\mathrm{SiO}}$, absorption is weak, and the resonances do not overlap, while in $\mathrm{W}$, the strong absorption leads to overlap of the Stokes and anti-Stokes resonances. Finally, the reflection coefficient $R$ modifies the overlap by reducing the amplitude of the Stokes resonance. The imaginary part of the Fano parameter reflects the energy lost during the reflection process through the acoustic reflection coefficient $R$.

We now investigate the experimental appearance of Fano resonances in the smooth $\mathrm{W}$ film. The transient reflectivity obtained at a pump and probe wavelength of $860 \mathrm{~nm}$ is represented in Fig. 2(a).

One first remarks an initial rise of the reflectivity produced by photogenerated carriers. A decay follows due to electron-electron and electron-phonon scattering. We also observe structures at 42 and $84 \mathrm{ps}$, called acoustic echoes. They correspond to the detection of the CAPs after one and two round-trips in the thin film, respectively. Considering
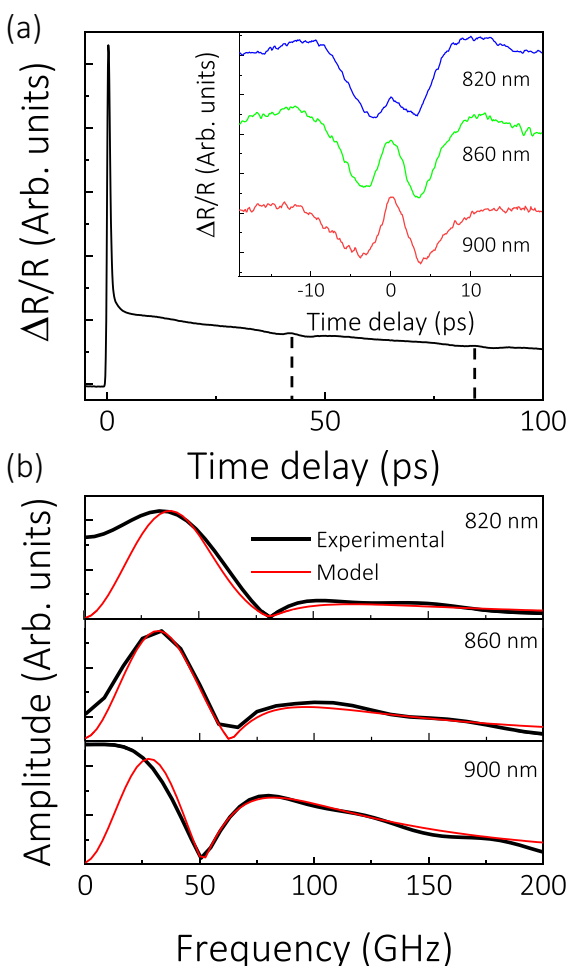

FIG. 2. Experimental investigation and modeling. (a) Transient reflectivity obtained on a 110-nm W film deposited on a Si substrate for a pump and probe wavelength of $860 \mathrm{~nm}$. Inset: Echoes obtained for a probe wavelength of 820 (blue), 860 (green), and $900 \mathrm{~nm}$ (red). (b) Fourier transform of the acoustic echoes (black lines) obtained at 820 (upper panel), 860 (middle panel), and $900 \mathrm{~nm}$ (lower panel) and model (red lines).

a longitudinal sound velocity of $5200 \mathrm{~m} / \mathrm{s}$ [38], we obtain a round-trip time of 42.3 ps. In the inset of Fig. 2(a), a zoom on the first echoes obtained for different wavelengths is shown. We see an oscillation growing in amplitude until $t=$ 0 , which corresponds to the CAPs propagating towards the surface. After $t=0$, the signal reverses due to the reflection of the CAPs and the following propagation towards the substrate. The echoes' shape is changing due to the variation of the optical and photo-elastic properties with the wavelength [30].

We now model the interaction of light with these CAPs using our analysis. In picosecond ultrasonic experiments, we measure the transient reflectivity, which is given by $\frac{\Delta R}{R_{0}} \simeq$ $2 \operatorname{Re}\left(\frac{\delta r}{r_{0}}\right)$. The spectrum of the transient reflectivity is thus given by the real part of the product of the scattering cross section [Eq. (4)] and the frequency content of the pulse. We consider the strain to be an odd function, which is a good approximation for CAPs generated at a free surface $[29,30,32]$. In that case, the spectrum of the strain is imaginary; the transient reflectivity spectrum is therefore proportional to the imaginary part of Eq. (4). Taking into account these considerations, we can calculate the spectra of these echoes for different wavelengths (see Supplemental Material [34]).

In Fig. 2(b), we show the Fourier transform for the echoes obtained at 820,860 , and $900 \mathrm{~nm}$. In each case, we observe a Fano-type line shape with a dip in the spectrum correspond- 

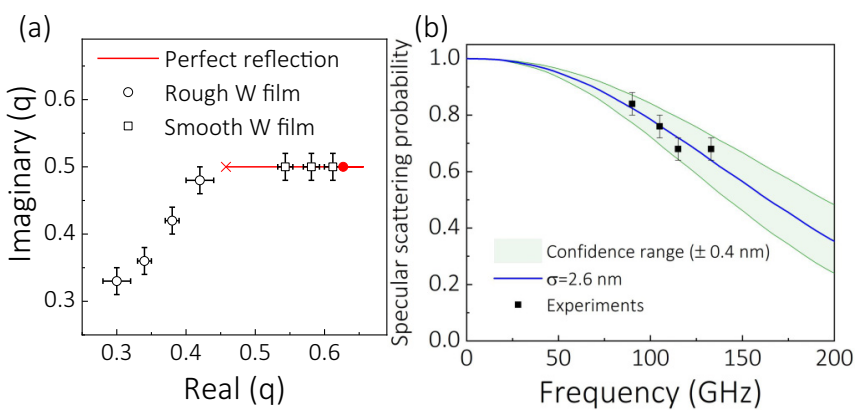

FIG. 3. Dissipation vs decoherence. (a) Real and imaginary parts of the Fano parameter for a smooth (squares) and rough $\mathrm{W}$ surface (circles) obtained experimentally at different probe wavelengths, and evolution of the real and imaginary parts of the Fano parameter for wavelengths ranging from 350 to $1000 \mathrm{~nm}$ for the case of a perfect reflection (red line). The beginning and ending of the wavelength range are marked by a red cross and circle, respectively. (b) Specular scattering probability extracting from the measurements of the Fano parameters and fit using the surface RMS roughness, with $\sigma$ as a fitting parameter.

ing to the destructive interferences. When the wavelength increases, the Fano line shape transforms: The frequency of the Fano minimum decreases and gets closer to the Fano maximum, corresponding to a decrease in the Fano parameters, in agreement with the ratio $n / \kappa$, which varies from 1.22 at $820 \mathrm{~nm}$ to 1.08 at $900 \mathrm{~nm}$. We also reproduce the spectra obtained using our model (see Supplemental Material [34]). Here, we used the refractive index values given in Ref. [37] and an acoustic reflection coefficient of -1 , corresponding to a perfect reflection of the CAPs. For the photoelastic coefficients, we used values from Ref. [30]. We observe an excellent agreement between our model and experimental data confirming that the transient reflectivity induced by the reflection of the CAP pulse can be modeled as a complex Fano resonance between the Stokes and anti-Stokes Brillouin scattering amplitudes. The differences at low frequencies come from the subtraction of the electronic contribution to the signal. Previous investigations observed such a dip in the acoustic echoes' spectrum [27,31,32], but their origin has not yet been connected to Fano resonances. We have applied our model to these observations and can reproduce these results (see Supplemental Material [34]), showing how general this phenomenon is.

We have demonstrated the possibility to observe Fano resonances due to the interference between the parametric emission and absorption of phonons by light. Now, we show how such engineered Fano resonance can be used to perform interface characterization. Previous work has shown that an analysis of the $q$ parameter trajectory in the complex plane reveals the origin of incoherent dynamics [13]. We use our model to extract the real and imaginary part of the $q$ parameter for the smooth and the rough $\mathrm{W}$ film for different probe wavelengths (see Supplemental Material [34]). In Fig. 3(a), we reproduce these trajectories.

We observe a striking difference between the trends for smooth and rough surfaces. On the one hand, for the smooth sample, only the real part, given by $R n / 2 \kappa$, is changing. The imaginary part, which represents losses and is proportional to
$R$, remains constant at 0.5 , which corresponds to $R=-1$ as expected for a smooth $\mathrm{W} /$ air interface. On the other hand, the Fano parameter's real and imaginary parts are simultaneously changing for the rough sample, which means that the acoustic reflection coefficient varies with the phonon frequency. To model this behavior, we introduce a modified reflection coefficient $\tilde{R}=R \cdot \operatorname{SSP}(\sigma)$, where $\mathrm{SSP}$ is the specular scattering probability that depends on $\sigma$, the root-mean-square (RMS) roughness of the sample (see Supplemental Material [34]) $[28,33,39]$. This model allows separating dissipation from decoherence: Dissipation corresponds to a change in the reflection coefficient, i.e., the energy is dissipated into another medium, which is independent of the phonon frequency, while decoherence at the interface would reduce the SSP, which is further reduced for high-frequency phonons. From the fitted Fano parameters, we extract the reflection coefficient $\tilde{R}$, and thus SSP at the frequency of the Fano interference, assuming that $R$ is the same as for the smooth surface case (see Supplemental Material [34]). In Fig. 3(b), we report the value of SSP extracted experimentally at different phonon frequencies, and a fit of the SSP using the lowest-order nonlocal small slope approximation with $\sigma$ as a fitting parameter [28,33,39]. We obtain $\sigma=2.6 \mathrm{~nm} \pm 0.4$. For comparison, we performed atomic force microscopy (AFM) measurements that revealed an RMS roughness of $1.8 \mathrm{~nm} \pm 0.15$ in relatively good agreement with our observation. The discrepancy may originate from the simplicity of our model, which neglects mode conversion, dissipation, and other losses at the interface. Nevertheless, our methods offer the possibility to nondestructively characterize the roughness of surfaces and buried interfaces.

In conclusion, we predict and model the complex Fano resonances resulting from interference in the scattering amplitude of light by coherent acoustic phonons reflecting at an interface. We highlight the conditions necessary for the appearance of such line shapes. We then experimentally verify their appearance by performing picosecond ultrasonic experiments on a thin $\mathrm{W}$ film. We obtain an excellent agreement between the experimental observations and our model. We also apply our model to other reports from the literature, highlighting the universality of this phenomenon. Finally, we demonstrate how we can dissociate dissipation from decoherence by monitoring the evolution of the complex Fano parameter as a function of wavelength. We then apply this principle to the nondestructive characterization of interface roughness, showing a good agreement with atomic force microscopy characterization. Our results can be extended, not only to other types of propagating waves that scatter light but also more generally to the interference between parametric emission and absorption. The observed Fano resonance and the enhanced sensitivity of such interferometric methods open the way for improved and novel characterizations, particularly for interface characterization and interfacial thermal transport in the case of phonons.

This work was supported by NanoLund, Lunds Universitet, Crafoordska Stiftelsen, Grant No. 20200630 and Vetenskapsrådet, Grant No. 2017-05150. The authors would like to thank Peter Blomqvist and Austin Irish for their help with AFM measurements. 
[1] U. Fano, Effects of configuration interaction on intensities and phase shifts, Phys. Rev. 124, 1866 (1961).

[2] H. Beutler, Über Absorptionsserien von Argon, Krypton und Xenon zu Termen zwischen den beiden Ionisierungsgrenzen ${ }^{2} \mathrm{P}_{3}^{2 / 0}$ und $^{2} \mathrm{P}_{1}^{2 / 0}$, Z. Phys. 93, 177 (1935).

[3] B. Lukyanchuk, N. I. Zheludev, S. A. Maier, N. J. Halas, P. Nordlander, H. Giessen, and C. T. Chong, The Fano resonance in plasmonic nanostructures and metamaterials, Nat. Mater. 9, 707 (2010).

[4] A. E. Miroshnichenko, S. Flach, and Y. S. Kivshar, Fano resonances in nanoscale structures, Rev. Mod. Phys. 82, 2257 (2010).

[5] B. Gallinet and O. J. F. Martin, Influence of electromagnetic interactions on the line shape of plasmonic Fano resonances, ACS Nano 5, 8999 (2011).

[6] W. Wang, Y. Jin, W. Wang, B. Bonello, B. Djafari-Rouhani, and R. Fleury, Robust Fano resonance in a topological mechanical beam, Phys. Rev. B 101, 024101 (2020).

[7] T. Still, W. Cheng, M. Retsch, R. Sainidou, J. Wang, U. Jonas, N. Stefanou, and G. Fytas, Simultaneous Occurrence of Structure-Directed and Particle-Resonance-Induced Phononic Gaps in Colloidal Films, Phys. Rev. Lett. 100, 194301 (2008).

[8] F. Lemoult, N. Kaina, M. Fink, and G. Lerosey, Wave propagation control at the deep subwavelength scale in metamaterials, Nat. Phys. 9, 55 (2013).

[9] T. Vasileiadis, H. Zhang, H. Wang, M. Bonn, G. Fytas, and B. Graczykowski, Frequency-domain study of nonthermal gigahertz phonons reveals Fano coupling to charge carriers, Sci. Adv. 6, eabd4540 (2020).

[10] S. Xiao, Y. Yoon, Y.-H. Lee, J. P. Bird, Y. Ochiai, N. Aoki, J. L. Reno, and J. Fransson, Detecting weak coupling in mesoscopic systems with a nonequilibrium Fano resonance, Phys. Rev. B 93, 165435 (2016).

[11] S. Zhang, K. Bao, N. J. Halas, H. Xu, and P. Nordlander, Substrate-induced Fano resonances of a plasmonic nanocube: A route to increased-sensitivity localized surface plasmon resonance sensors revealed, Nano Lett. 11, 1657 (2011).

[12] A. A. Clerk, X. Waintal, and P. W. Brouwer, Fano Resonances as a Probe of Phase Coherence in Quantum Dots, Phys. Rev. Lett. 86, 4636 (2001).

[13] A. Bärnthaler, S. Rotter, F. Libisch, J. Burgdörfer, S. Gehler, U. Kuhl, and H.-J. Stöckmann, Probing Decoherence through Fano Resonances, Phys. Rev. Lett. 105, 056801 (2010).

[14] D. Finkelstein-Shapiro and A. Keller, Ubiquity of Beutler-Fano profiles: From scattering to dissipative processes, Phys. Rev. A 97, 023411 (2018).

[15] M. I. Tribelsky and A. E. Miroshnichenko, Giant in-particle field concentration and Fano resonances at light scattering by high-refractive-index particles, Phys. Rev. A 93, 053837 (2016).

[16] U. Siegner, M. A. Mycek, S. Glutsch, and D. S. Chemla, Ultrafast Coherent Dynamics of Fano Resonances in Semiconductors, Phys. Rev. Lett. 74, 470 (1995).

[17] M. Wickenhauser, J. Burgdörfer, F. Krausz, and M. Drescher, Time Resolved Fano Resonances, Phys. Rev. Lett. 94, 023002 (2005).

[18] C. Ott, A. Kaldun, P. Raith, K. Meyer, M. Laux, J. Evers, C. H. Keitel, C. H. Greene, and T. Pfeifer, Lorentz meets Fano in spectral line shapes: A universal phase and its laser control, Science 340, 716 (2013).
[19] A. Kaldun, A. Blättermann, V. Stooß, S. Donsa, H. Wei, R. Pazourek, S. Nagele, C. Ott, C. D. Lin, J. Burgdörfer, and T. Pfeifer, Observing the ultrafast buildup of a Fano resonance in the time domain, Science 354, 738 (2016).

[20] M. Kotur, D. Guénot, Á. Jiménez-Galán, D. Kroon, E. W. Larsen, M. Louisy, S. Bengtsson, M. Miranda, J. Mauritsson, C. L. Arnold, S. E. Canton, M. Gisselbrecht, T. Carette, J. M. Dahlström, E. Lindroth, A. Maquet, L. Argenti, F. Martín, and A. L'Huillier, Spectral phase measurement of a Fano resonance using tunable attosecond pulses, Nat. Commun. 7, 10566 (2016).

[21] M. I. Tribelsky and A. E. Miroshnichenko, Dynamics of destructive Fano resonances, Phys. Rev. A 100, 053824 (2019).

[22] L. Brillouin, Diffusion de la lumière et des rayons x par un corps transparent homogène - influence de l'agitation thermique, Ann. Phys. (Paris) 9, 88 (1922).

[23] A. Borovik-Romanov and N. Kreines, Brillouin-Mandelstam scattering from thermal and excited magnons, Phys. Rep. 81, 351 (1982)

[24] P. Y. Yu, Brillouin scattering of exciton polaritons and additional boundary conditions, Solid State Commun. 32, 29 (1979).

[25] N. T. Otterstrom, R. O. Behunin, E. A. Kittlaus, Z. Wang, and P. T. Rakich, A silicon Brillouin laser, Science 360, 1113 (2018).

[26] G. Bahl, M. Tomes, F. Marquardt, and T. Carmon, Observation of spontaneous Brillouin cooling, Nat. Phys. 8, 203 (2012).

[27] P.-A. Mante, A. Devos, and A. Le Louarn, Generation of terahertz acoustic waves in semiconductor quantum dots using femtosecond laser pulses, Phys. Rev. B 81, 113305 (2010).

[28] P.-A. Mante, C.-C. Chen, Y.-C. Wen, J.-K. Sheu, and C.-K. Sun, Thermal Boundary Resistance between GaN and Cubic Ice and $\mathrm{THz}$ Acoustic Attenuation Spectrum of Cubic Ice from Complex Acoustic Impedance Measurements, Phys. Rev. Lett. 111, 225901 (2013).

[29] C. Thomsen, H. T. Grahn, H. J. Maris, and J. Tauc, Surface generation and detection of phonons by picosecond light pulses, Phys. Rev. B 34, 4129 (1986).

[30] A. Devos and C. Lerouge, Evidence of Laser-Wavelength Effect in Picosecond Ultrasonics: Possible Connection with Interband Transitions, Phys. Rev. Lett. 86, 2669 (2001).

[31] C. He, O. Ristow, M. Grossmann, D. Brick, Y. Guo, M. Schubert, M. Hettich, V. Gusev, and T. Dekorsy, Acoustic waves undetectable by transient reflectivity measurements, Phys. Rev. B 95, 184302 (2017).

[32] O. Matsuda, M. C. Larciprete, R. Li Voti, and O. B. Wright, Fundamentals of picosecond laser ultrasonics, Ultrasonics 56, 3 (2015).

[33] Y.-C. Wen, C.-L. Hsieh, K.-H. Lin, H.-P. Chen, S.-C. Chin, C.-L. Hsiao, Y.-T. Lin, C.-S. Chang, Y.-C. Chang, L.-W. Tu, and C.-K. Sun, Specular Scattering Probability of Acoustic Phonons in Atomically Flat Interfaces, Phys. Rev. Lett. 103, 264301 (2009).

[34] See Supplemental Material at http://link.aps.org/supplemental/ 10.1103/PhysRevResearch.3.L032010 for detailed calculations of the model, a comparison of the model to experiments in the literature, AFM images of the rough surface, and fitting of the model to the experimental data obtained on the rough sample.

[35] L. V. R. de Marcos, J. I. Larruquert, J. A. Méndez, and J. A. Aznárez, Self-consistent optical constants of $\mathrm{SiO}_{2}$ and $\mathrm{Ta}_{2} \mathrm{O}_{5}$ films, Opt. Mater. Express 6, 3622 (2016). 
[36] P. A. Mante, J. F. Robillard, and A. Devos, Complete thin film mechanical characterization using picosecond ultrasonics and nanostructured transducers: experimental demonstration on $\mathrm{SiO}_{2}$, Appl. Phys. Lett. 93, 071909 (2008).

[37] A. D. Rakić, A. B. Djurišić, J. M. Elazar, and M. L. Majewski, Optical properties of metallic films for vertical-cavity optoelectronic devices, Appl. Opt. 37, 5271 (1998).
[38] G. V. Samsonov, Handbook of the Physicochemical Properties of the Elements (Springer, New York, 1968).

[39] S. L. Broschat, Reflection loss from a "Pierson-Moskowitz" sea surface using the nonlocal small slope approximation, IEEE Trans. Geosci. Remote Sens. 37, 632 (1999). 